\title{
Effect of Chloroform on the Regenerating Renal Tubular Epithelium in Mouse
}

Subcutaneous administration of chloroform to male mice of various strains results in necrosis of considerable segments of renal proximal convoluted tubules. This lesion is fully developed 2 days after injection, but does not cause the death of the animal. The majority of strains (except $\mathrm{C} 3 \mathrm{H} / \mathrm{He}$ ) develop tubular regeneration ${ }^{1,2}$. Harmful effect seems to depend upon the absorption of a metabolite of chloroform by the epithelium.

In order to get some more information to shed light on the possible pathomechanism of the action of chloroform, we checked the effect of this substance on the epithelium which regenerates after the 1 st dose of the drug. We assumed that the possible effect of 2 nd dose of chloroform may be expected in the later phase of regeneration when the epithelium is morphologically more similar to the normal one, and the restoration of tubular absorbing capacity more probable.

Material and method. The experiment was carried out on 30 male mice of the generation F1 (o C57BL/6JN $x$ $\approx \mathrm{C} 3 \mathrm{H} / \mathrm{He}$ ). Each mouse was injected s.c. with a single dose of $0.1 \mathrm{ml}$ of the solution: $0.05 \mathrm{~g}$ of chloroform in $1 \mathrm{ml}$

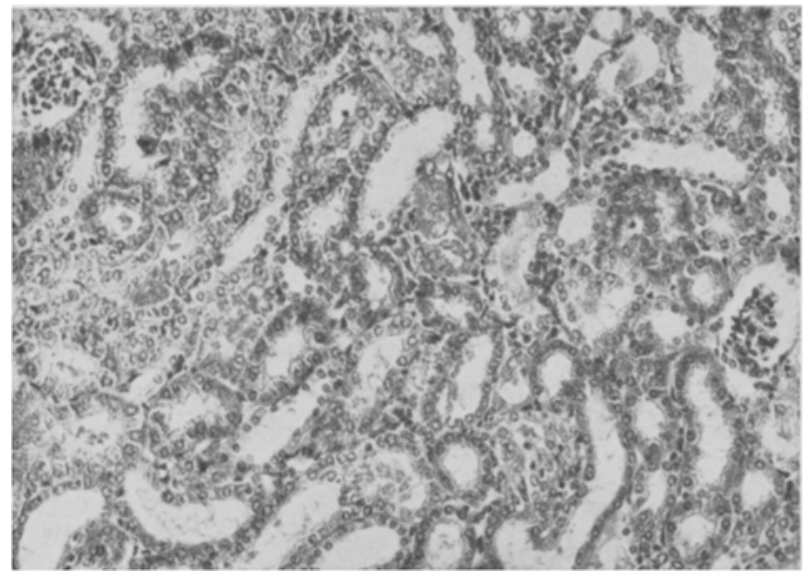

Fig. 1. Mouse kidney 8 days after the injection of the 1st dose of chloroform. Marked regeneration of the epithelium of proximal convoluted tubules. The epithelial cells are still immature, lower than normal, and fail to stain well with eosin. H. E.; $\times 100$.

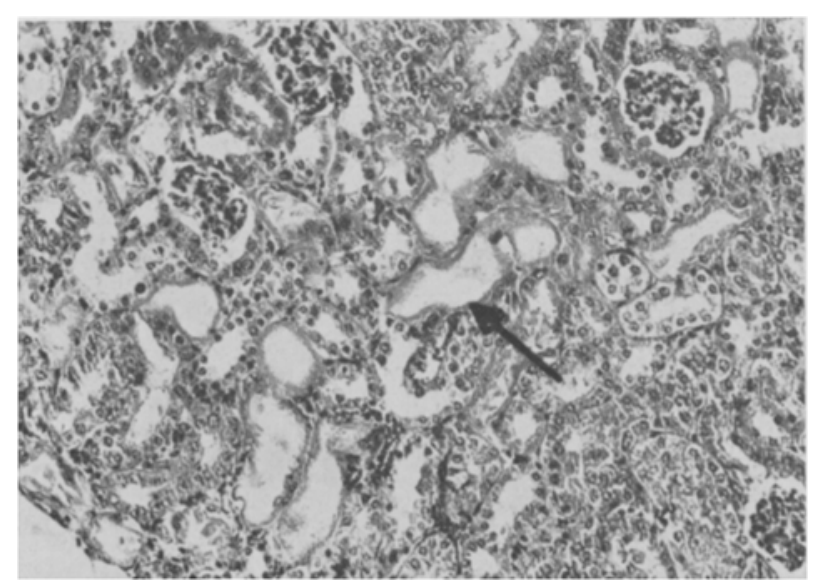

Fig. 2. Mouse kidney 10 days after injection of the 1 st dose of chloroform and 2 days after injection of the 2 nd dose. Segmental necrosis of tubular epithelium (arrow), acidophilic in original section. H. E.; $\times 100$. of ethyl laurate. On the 2nd, 4th, 6th, 8 th, 10 th and 14 th day after the 1 st chloroform injection 1 mouse was killed in order to evaluate the tubular epithelium (control), and 4 mice were again treated with chloroform as mentioned above. Out of these 4 mice two were killed 2 days, and two 14 days after the 2nd chloroform injection. Paraffin sections of the kidneys were stained with hematoxylin and eosin, as well as with PAS.

Results. All mice survived 1 or 2 doses of chloroform. 2 days after the 1st injection there was an extensive necrosis of proximal convoluted tubules. The 2 nd dose of chloroform failed to change the renal appearance after 2 further days.

On the 4 th and 6 th day after the 1st chloroform dose, the control mice showed ovbious regeneration. The brush border was present in the epithelium of some tubular cross-sections, the majority of them being devoid of it, however. 2 days after the injection of the 2 nd dose, there was no difference in tubular morphology.

On the 8th, 10th and 14th day after the injection of 1st dose of chloroform, the regeneration was far advanced (Figure 1), and the regenerating epithelium showed the brush border on many cross-sections. 2 days after the injection of 2nd dose some tubular segments were necrotic (Figure 2). The necrotic epithelium showed the brush border. Necrotic segments were very few, and the lesion was much less extensive than that observed 2 days after the 1 st chloroform dose. Locally, the necrotic epithelium was already undermined by the newly regenerating one.

All mice which survived up to the 14th day after the 2nd chloroform injection showed tubular regeneration.

Discussion. Up to the 6th day after injection of the 1 st dose of chloroform, the regenerating epithelium of proximal convolutions seems to be insensitive to the 2nd dose of this substance. Segmental tubular necrosis was found, however, when the 2nd dose was administred on the 8 th, 10 th and 14 th day of regeneration.

The brush border can be considered as the morphological index of absorption ability of the tubular cell. Within the first 6 days it is present only on a few crosssections of the regenerating tubules, but is much more extensive on the 8 th, 10 th and 14 th day. This fact could justify the presence of tubular necrosis provided that the latter results from the absorption of the harmful substance. Necrosis is, however, much less extensive than that found after the 1st chloroform injection. This seems to depend as well upon the lesser maturity of the regenerating epithelium in comparison with the normal one as upon its much greater readiness to secondary regenerative response.

Résumé. Chez la souris, après l'injection de la première dose de chloroforme, la seconde dose entraîne la nécrose segmentaire de l'épithélium tubulaire rénal, depuis le huitième jour de sa régénération. Après la seconde dose, la nécrose tubulaire a une moins grande extension.

\section{S. KRUŚ and Z. ZALESKA-RUTCZYÁSKA}

Department of Pathological Anatomy,

Department of Histology and Embryology, Institute of Biostructure, Medical Academy, Chatubinskiego 5, Warsaw (Poland), 12 June 1972.

1 S. KRuś and Z. ZaLESKA-RUTCZYŃSKA, Experientia 26, 101 (1970). 2 S. KRUŚ and Z. ZALESKA-RUTCZYŃSKA, Experientia 26, 750 (1970) 